MODELING, IDENTIFICATION AND CONTROL, 1994, VOL. 15, NO. 2, 81-91 doi:10.4173/mic.1994.22

\title{
Cascaded adaptive control of ocean vehicles with significant actuator dynamics
}

\author{
THOR I. FOSSEN† and OLA-ERIK FJELLSTAD†
}

Keywords: Adaptive control, actuator dynamics, ship steering, ROV.

\begin{abstract}
This paper presents a cascade adaptive control scheme for marine vehicles where the non-linear equations of motion include a model of the actuator dynamics. The adaptive controller does not require the parameters of the vehicle dynamics and the actuator time constants to be known a priori. Both the velocity and position tracking errors are shown to converge to zero by applying Barbălat's lemma. Global asymptotic stability is proven for the velocity scheme while the position/attitude controller is only proven to be convergent. Furthermore, all parameter estimates are shown to be bounded. Computer simulations of an ROV speed control system and an autopilot for automatic ship steering are used to illustrate the design methodology.
\end{abstract}

\section{Introduction}

Adaptive control theory have been successfully applied to marine vehicles such as underwater vehicles, submersibles and surface ships. However, most of these methods are based on the assumption that the actuator dynamics can be ignored in the control design. Including a model of the actuator can improve the robustness and performance of the control system. Fjellstad et al. (1992) propose to use the reference model decomposition (RMD) technique of Butler et al. (1991) to compensate for the unmodeled underwater vehicle thruster dynamics. However, global stability has not been proven for this method. This paper presents a new globally stable adaptive controller for marine vehicles where a model of the actuator dynamics is included. Consider the 6 degrees of freedom (DOF) nonlinear ocean vehicle equations of motion in the form:

$$
\begin{array}{ll}
\text { Vehicle dynamics } & M \dot{v}+C(v) v+D(v) v+g(\eta)=B(v) u \\
\text { Kinematics } & \dot{\eta}=J(\eta) v \\
\text { Actuator dynamics } & T \dot{\boldsymbol{u}}+\boldsymbol{u}=u_{c}
\end{array}
$$

where $\boldsymbol{v}=(u, v, w, p, q, r)^{\mathrm{T}}$ is a vector of body-fixed linear and angular velocity components, and $\boldsymbol{\eta}=(x, y, z, \phi, \theta, \psi)^{\mathrm{T}}$ is a vector of positions $(x, y, z)$ and Euler angles $(\phi, \theta, \psi)$. The components $(u, v, w, p, q, r)$ and $(x, y, z, \phi, \theta, \psi)$ correspond to the 6 DOF motion variables in surge, sway, heave, roll, pitch and yaw. $u \in \mathbb{R}^{p}(p \geqslant 6)$ is a vector of actual control inputs, and $\boldsymbol{u}_{c} \in \mathbb{R}^{p}$ is a vector of commanded actuator inputs. $\boldsymbol{M}$ is the unknown inertia matrix (including hydrodynamic inertia), $C(v)$ is an unknown matrix

Received 6 May 1993.

† Department of Engineering Cybernetics. The Norwegian Institute of Technology, N-7034 Trondheim, Norway.

Reprinted from the Preprints of the 12th IFAC World Congress, Sydney, 18-23 July 1993, with kind permission from Pergamon Press I.td, Headington Hill Hall, Oxford OX3 OBW, UK. 
of Coriolis and centripetal terms (including hydrodynamic added mass coupling terms), $\boldsymbol{D}(\boldsymbol{v})$ is an unknown matrix representing the hydrodynamic damping terms, $\boldsymbol{g}(\boldsymbol{\eta})$ is an unknown vector of restoring forces and moments, while $B(v)$ is a known $6 \times p$ input matrix. $J(\eta)$ is a $6 \times 6$ known block diagonal transformation matrix relating the bodyfixed reference frame to the inertial reference frame (usually the earth). Notice that $\boldsymbol{J}(\boldsymbol{\eta})$ depends on the Euler angles $(\phi, \theta, \psi)$ only, while $\boldsymbol{g}(\eta)$ depends on $\phi$ and $\theta . \boldsymbol{T}=\operatorname{diag}\left\{t_{i}\right\}$ is a $p \times p$ diagonal matrix of positive unknown actuator time constants $\left(t_{i}>0\right)$. The different matrices can be shown to satisfy the following properties.

Property $1(M)$

For a rigid-body moving with low speed through an ideal fluid, the added inertia matrix is positive definite, that is

$$
\boldsymbol{M}=\boldsymbol{M}^{\mathrm{T}}>0
$$

In a real fluid the 36 elements of $M$ may all be distinct. However, experience has shown that the numerical values of the added mass derivatives in a real fluid usually correspond well with those obtained from ideal theory.

Proof: See Newman (1977).

Property $2(C)$

For a rigid-body moving through an ideal fluid the Coriolis and centripetal forces: $C(v) v$ can always be parameterized such that $C(v)$ is skew-symmetrical, that is

$$
C(v)=-C^{\mathrm{T}}(v) \forall v \in \mathbb{R}^{6}
$$

which implies that

$$
x^{\mathrm{T}} C(v) x=0 \forall v, x \in \mathbb{R}^{6}
$$

Proof. A skew-symmetric representation of $C(v)$ can be derived from the vector form of Kirchhoff's equations of motion (Kirchhoff 1896). The complete proof is found in Sagatun and Fossen (1991).

Property $3(D)$

The hydrodynamic damping matrix $D(v)$ is positive, that

$$
D(v)>0 \forall v \in \mathbb{R}^{6}, v \neq 0
$$

Proof. This is obvious since all ocean vehicles are dissipative.

For ocean vehicles, $\boldsymbol{M}$ will depend on the frequency of encounter between the vehicle and the waves and thus the speed of the vehicle. This relationship is non-trivial for a vehicle moving in 6 DOF. For simplicity, we will assume that $\dot{\boldsymbol{M}}=0$ for each sea condition.

The paper is outlined as follows. Section 2 presents the control scheme when all the parameters are known, while the adaptive control law is derived in Section 3. In Section 4 computer simulations of an ROV speed control system and an autopilot for automatic ship steering are used to illustrate the design methodology. Our conclusions are given at the end of the paper. 


\section{Globally asymptotically stable control of ocean vehicles}

Let us initially assume that $\boldsymbol{M}, \boldsymbol{C}(v), D(v), g(\eta), T$ and $B(v)$ are known. Consider the Lyapunov function candidate

$$
V(\tilde{\boldsymbol{v}}, \tilde{\boldsymbol{u}})=\frac{1}{2}\left(\tilde{\boldsymbol{v}}^{\mathrm{T}} \boldsymbol{M} \tilde{\boldsymbol{v}}+\tilde{\boldsymbol{u}}^{\mathrm{T}} \boldsymbol{T} \tilde{\boldsymbol{u}}\right)
$$

where $\tilde{\boldsymbol{v}}=\boldsymbol{v}-\boldsymbol{v}_{d}$ and $\tilde{\boldsymbol{u}}=\boldsymbol{u}-\boldsymbol{u}_{d}$ are the tracking errors and $\boldsymbol{v}_{d} \in C^{2}$ and $\boldsymbol{u}_{d} \in C^{1}$ are the desired velocity and actuator state output, respectively. Differentiating $V$ with respect to time and applying Property 1 and 2, yields (assuming $\dot{M}=0$ )

$$
\dot{V}=\tilde{v}^{\mathrm{T}}[M \dot{\tilde{v}}+C(v) \tilde{v}]+\tilde{u}^{\mathrm{T}} T \dot{\tilde{u}}
$$

Hence the substituting of (1) and (3) into the expression for $\dot{V}$, yields

$$
\begin{aligned}
\dot{V}= & \tilde{v}^{\mathrm{T}}\left[M \dot{v}+C(v) v-M \dot{v}_{d}-C(v) v_{d}\right] \\
& +\tilde{u}^{\mathrm{T}}\left[T \dot{u}-T \dot{u}_{d}\right] \\
= & -\tilde{v}^{\mathrm{T}} D(v) \tilde{v}+\tilde{v}^{\mathrm{T}}\left[B(v) u-M \dot{v}_{d}-C(v) v_{d}\right. \\
& \left.-D(v) v_{d}-g(\eta)\right]+\tilde{u}^{\mathrm{T}}\left[u_{c}-u-T \dot{u}_{d}\right]
\end{aligned}
$$

Assuming that $\boldsymbol{M}, \boldsymbol{C}(\boldsymbol{v}), \boldsymbol{D}(\boldsymbol{v}), \boldsymbol{g}(\boldsymbol{\eta})$ are linear in their parameters, the parameter vector $\theta$ and its corresponding regression matrix $\Phi\left(\dot{v}_{d}, v_{d}, v, \eta\right)$ can be derived from

$$
M \dot{v}_{d}+C(v) v_{d}+D(v) v_{d}+g(\eta) \triangleq \Phi\left(\dot{v}_{d}, v_{d}, v, \eta\right) \theta
$$

Notice, that $\Phi\left(\dot{v}_{d}, v_{d}, v, \eta\right)$ is a known matrix function of the measured states $v$ and $\eta$ (in fact, only the components $\phi$ and $\theta$ (roll and pitch) of $\eta$ must be measured). Applying this parameterization to $\dot{V}$ yields

$$
\dot{V}=-\tilde{v}^{\mathrm{T}} D(v) \tilde{v}+\tilde{v}^{\mathrm{T}}\left[B(v) u-\Phi\left(\dot{v}_{d}, v_{d}, v, \eta\right) \theta\right]+\tilde{u}^{\mathrm{T}}\left[u_{c}-u-T \dot{u}_{d}\right]
$$

Since, $\tilde{v}^{\mathrm{T}} \boldsymbol{B}(\boldsymbol{v}) \tilde{\boldsymbol{u}}=\tilde{\boldsymbol{u}}^{\mathrm{T}} \boldsymbol{B}^{\mathrm{T}}(\boldsymbol{v}) \tilde{\boldsymbol{v}}$, we can subtract the vector: $\boldsymbol{B}(\boldsymbol{v}) \tilde{\boldsymbol{u}}=\boldsymbol{B}(\boldsymbol{v}) \boldsymbol{u}-\boldsymbol{B}(\boldsymbol{v}) \boldsymbol{u}_{d}$ from the first bracket and add the vector: $\boldsymbol{B}^{\mathrm{T}}(\boldsymbol{v}) \tilde{v}$ to the second bracket. Consequently,

$$
\dot{V}=-\tilde{v}^{\mathrm{T}} D(v) \tilde{v}+\tilde{v}^{\mathrm{T}}\left[B(v) u_{d}-\Phi\left(\dot{v}_{d}, v_{d}, v, \eta\right) \theta\right]+\tilde{u}^{\mathrm{T}}\left[u_{c}-u-T \dot{u}_{d}+B^{\mathrm{T}}(v) \tilde{v}\right]
$$

The control objective can be defined as

$$
\lim _{t \rightarrow \infty} \tilde{\boldsymbol{v}}(t)=0 ; \quad \lim _{t \rightarrow \infty} \tilde{u}(t)=0
$$

which simply states that the velocity tracking error $\tilde{\boldsymbol{v}}$ the actuator state tracking error $\tilde{\boldsymbol{u}}$ should converge to zero as $t=\infty$. This objective can be satisfied by applying the following lemma.

Lemma 1 (Globally asymptotically stable velocity control)

Consider the system (1), (2) and (3) and the following control law

$$
\boldsymbol{u}_{c}=\boldsymbol{u}+\boldsymbol{T} \dot{\boldsymbol{u}}_{d}-\boldsymbol{B}^{\mathrm{T}}(\boldsymbol{v}) \tilde{\boldsymbol{v}}-\boldsymbol{K}_{u} \tilde{u} ; \quad \boldsymbol{K}_{u}>0
$$

where $\boldsymbol{u}_{d}$ is computed from

$$
u_{d}=B^{+}(v)\left[\Phi\left(\dot{v}_{d}, v_{d}, v, \eta\right) \theta-K_{v} \tilde{v}\right] ; \quad K_{v} \geqslant 0
$$


Here $B^{+}(v)$ is the right Moore-Penrose $\dagger$ pseudo-inverse of $\boldsymbol{B}(v)$. Then the signals $\tilde{\boldsymbol{u}}$ and $\tilde{v}$ converge to zero as $t \rightarrow \infty$.

Proof. Substituting (11) and (12) into (9) and using the fact that: $\boldsymbol{B}(v) \boldsymbol{B}^{+}(v)=\boldsymbol{I}$, yields

$$
\dot{V}=-\tilde{v}^{\mathrm{T}}\left[K_{v}+D(v)\right] \tilde{v}-\tilde{u}^{\mathrm{T}} K_{u} \tilde{u} \leqslant 0
$$

This implies that $V(t) \leqslant V(0) \forall t \geqslant 0$, and accordingly that $\tilde{\boldsymbol{v}}$ and $\tilde{\boldsymbol{u}}$ are bounded. Furthermore $\dot{\hat{\boldsymbol{v}}}$ and $\dot{\tilde{\boldsymbol{u}}}$ is bounded, which implies that $\dot{\boldsymbol{V}}$ is uniformly continuous. Applications of Barbălat's lemma (Barbălat 1959 or Popov 1973) ensures that $\dot{V} \rightarrow 0$ and consequently that $\tilde{\boldsymbol{v}} \rightarrow 0$ and $\tilde{\boldsymbol{u}} \rightarrow 0$ as $t \rightarrow \infty$, which concludes the proof.

In the implementation of the control law (11) and (12), $\dot{u}_{d}$ must be computed. This implies that acceleration $\dot{\boldsymbol{v}}$, velocity $\boldsymbol{v}$ and the two Euler angles $\phi$ and $\theta$ must be measured or obtained through state estimation. In fact, global asymptotic stability is guaranteed even for $K_{v}=0$ since: $x^{\mathrm{T}} D(v) x>0 \forall v, x \in \mathbb{R}^{6}, x \neq 0 v \neq 0$ (Property 3). It seems reasonable to choose the maximum singular values of the gain matrices $K_{u}$ and $K_{v}$ according to

$$
\bar{\sigma}\left(\boldsymbol{K}_{u}\right)>\bar{\sigma}\left(\boldsymbol{K}_{v}\right)
$$

where $\bar{\sigma}(\cdot)$ is the maximum singular value, to ensure that the bandwidth of the inner servo loop (actuator dynamics) will be higher than the bandwidth of the outer loop (vehicle dynamics).

\section{Remark 1 (Negligible actuator dynamics)}

If the actuator is fast compared to the vehicle dynamics, it follows that $\boldsymbol{T}=0$ and consequently that $\boldsymbol{u}=\boldsymbol{u}_{\boldsymbol{c}}$. This implies that (4) reduces to

$$
V(\tilde{\boldsymbol{v}})=\frac{1}{2} \tilde{\boldsymbol{v}}^{\mathrm{T}} \boldsymbol{M} \tilde{\boldsymbol{v}}
$$

Differentiating this expression with respect to time suggests that the control law should be chosen as

to yield $\dot{V} \leqslant 0$.

$$
\boldsymbol{u}=\boldsymbol{B}^{+}(\boldsymbol{v})\left[\Phi\left(\dot{\boldsymbol{v}}_{d}, \boldsymbol{v}_{d}, \boldsymbol{v}, \boldsymbol{\eta}\right) \boldsymbol{\theta}-\boldsymbol{K}_{\boldsymbol{v}} \tilde{v}\right] ; \quad K_{v} \geqslant 0
$$

\section{Adaptive control of ocean vehicles}

In this section we will first derive a globally asymptotically stable adaptive controller for velocity control (Section 3.1) and then extend these results to a convergent adaptive position/attitude scheme (Section 3.2). Furthermore, we will assume that: $\boldsymbol{M}, \boldsymbol{C}(v), D(v), g(\eta)$ and $T$ are unknown and that the input matrix $\boldsymbol{B}(v)$ is known. The symbolic expression for $J(\eta)$ is also known a priori since equation (2) simply describes the orientation of the body-fixed reference frame relative to the inertial reference frame in terms of the Euler angles.

\subsection{Globally asymptotically stable adaptive velocity control}

Consider the modified Lyapunov function candidate

$$
V=\frac{1}{2}\left[\tilde{v}^{\mathrm{T}} \boldsymbol{M} \tilde{\boldsymbol{v}}+\tilde{u}^{\mathrm{T}} \boldsymbol{T} \tilde{u}+\tilde{\boldsymbol{\theta}}^{\mathrm{T}} \boldsymbol{\Gamma}^{-1} \tilde{\boldsymbol{\theta}}+\sum_{i=1}^{6} \frac{1}{\alpha_{i}} \tilde{t}_{i}^{2}\right]
$$

$\dagger \boldsymbol{B}^{+}=\boldsymbol{B}^{\mathrm{T}}\left(\boldsymbol{B} \boldsymbol{B}^{\mathrm{T}}\right)^{-1}$ is called the right Moore-Penrose pseudo-inverse of $\boldsymbol{B}$ which exists if the matrix $\boldsymbol{B} \boldsymbol{B}^{\mathrm{T}}$ is non-singular. Notice that the right pseudo-inverse $\boldsymbol{B}^{+}$has the property that $\boldsymbol{B B}^{+}=\boldsymbol{I}$. 
where $\Gamma=\Gamma^{\mathrm{T}}>0$ and $\alpha_{i}>0(i=1 \ldots 6)$. The parameter errors are defined as

$$
\tilde{\theta}=\hat{\theta}-\theta, \tilde{t_{i}}=\hat{t_{i}}-t_{i} ; \quad i=1 \ldots 6
$$

Theorem 1 (Globally asymptotically stable adaptive velocity control)

Consider the system (1), (2) and (3) and the following control law

$$
\begin{array}{lll}
\boldsymbol{u}_{d}=\boldsymbol{B}^{+}(\boldsymbol{v})\left[\Phi\left(\dot{v}_{d}, v_{d}, v, \eta\right) \hat{\boldsymbol{\theta}}-K_{v} \tilde{v}\right] ; & K_{v} \geqslant 0 \\
\boldsymbol{u}_{c}=\boldsymbol{u}+\hat{\boldsymbol{T}} \dot{u}_{d}-\boldsymbol{B}^{\mathrm{T}}(\boldsymbol{v}) \tilde{v}-K_{u} \tilde{u} ; & K_{u}>0
\end{array}
$$

with the parameter adaptation laws

$$
\begin{aligned}
& \hat{\boldsymbol{\theta}}=-\boldsymbol{\Gamma} \Phi^{\mathrm{T}}\left(\dot{\boldsymbol{v}}_{d}, \boldsymbol{v}_{d}, \boldsymbol{v}, \boldsymbol{\eta}\right) \tilde{\boldsymbol{v}} ; \quad \boldsymbol{\Gamma}>0 \\
& \dot{\hat{t}}_{i}=-\alpha_{i} \tilde{u}_{i} \dot{u}_{d i} ; \quad \alpha_{i}>0, \quad i=1 \ldots 6
\end{aligned}
$$

Then the signals $\tilde{\boldsymbol{\theta}}$ and $\tilde{\boldsymbol{T}}$ remain bounded and $\tilde{\boldsymbol{u}}$ and $\tilde{\boldsymbol{v}}$ converge to zero as $t \rightarrow \infty$ Proof. The proof is found in Appendix A.

\section{Remark 2 (Negligible actuator dynamics)}

If the actuator is fast compared to the vehicle dynamics, we have that $\boldsymbol{u}=\boldsymbol{u}_{\boldsymbol{c}}$ in view of $T=0$. Hence, the Lyapunov function candidate (17) reduces to

$$
V=\frac{1}{2}\left[\tilde{v}^{\mathrm{T}} \boldsymbol{M} \boldsymbol{v}+\hat{\boldsymbol{\theta}}^{\mathrm{T}} \boldsymbol{\Gamma}^{-1} \hat{\boldsymbol{\theta}}\right]
$$

which suggests that the adaptive control law should be expressed as

$$
u=B^{+}(v)\left[\Phi\left(\dot{v}_{d}, v_{d}, v, \eta\right) \hat{\theta}-K_{v} \tilde{v}\right]
$$

with the parameter update law (21) to yield $V \leqslant 0$. Notice that for $\boldsymbol{B}(v)=I$, this result is equivalent to the adaptive control law of Slotine and $\mathrm{Li}(1987)$ for robot manipulators.

\subsection{Convergent stable adaptive position/attitude control}

Assume that $\theta \neq \pm 90^{\circ}$ which implies that $\boldsymbol{J}^{-1}(\boldsymbol{\eta})$ exists. Hence, the vehicle's position and orientation can be controlled by noticing that (1) and (2) can be rewritten as

$$
M_{\eta}(\eta) \ddot{\eta}+C_{\eta}(v, \eta) \dot{\eta}+D_{\eta}(v, \eta) \dot{\eta}+g_{\eta}(\eta)=J^{-\mathrm{T}} B(v) u
$$

where

$$
\begin{aligned}
M_{\eta}(\eta) & =J^{-\mathrm{T}}(\eta) M J^{-1}(\eta) \\
C_{\eta}(v, \eta) & =J^{-\mathrm{T}}(\eta)\left[C(v) J^{-1}(\eta)+M \dot{J}^{-1}(\eta)\right] \\
D_{\eta}(v, \eta) & =J^{-\mathrm{T}}(\eta) D(v) J^{-1}(\eta) \\
g_{\eta}(\eta) & =J^{-\mathrm{T}}(\eta) g(\eta)
\end{aligned}
$$

The system (25) satisfies the following properties.

Property $4\left(M_{\eta}\right)$

The inertia matrix is symmetrical and positive definite, that is

$$
\boldsymbol{M}_{\eta}(\boldsymbol{\eta})=\boldsymbol{M}_{\eta}^{\mathrm{T}}(\boldsymbol{\eta})>0 \forall \boldsymbol{\eta} \in \mathbb{R}^{6}, \theta \neq \pm 90^{\circ}
$$

Proof. Follows directly from Property 1. 
Property $5\left(D_{\eta}\right)$

The damping matrix $D_{\eta}(v, \eta)$ is positive, that is

$$
D_{\eta}(v, \eta)>0 \forall \eta, v \in \mathbb{R}^{6}, \theta \neq \pm 90^{\circ}
$$

Proof. Follows directly from Property 3.

Property $6\left(\dot{M}_{\eta}-2 C_{\eta}\right)$

The matrix $\left(\boldsymbol{M}_{\eta}-2 \boldsymbol{C}_{\eta}\right)$ is skew-symmetrical, which implies that the following holds

$$
x^{\mathrm{T}}\left[\dot{M}_{\eta}(\eta)-2 C_{\eta}(v, \eta)\right] x=0 \forall \eta, v, x \in \mathbb{R}^{6}
$$

Proof. See Fossen (1994).

Notice that, $\boldsymbol{C}_{\eta} \neq \boldsymbol{C}_{\eta}^{\mathrm{T}}$ and $\dot{\boldsymbol{M}}_{\eta} \neq 0$. Consider the modified Lyapunov function candidate

$$
V=\frac{1}{2}\left[s^{\mathrm{T}} M_{\eta} s+\tilde{u}^{\mathrm{T}} T \tilde{u}+\tilde{\theta}^{\mathrm{T}} \Gamma^{-1} \tilde{\theta}+\sum_{i=1}^{6} \frac{1}{\alpha_{i}} \tilde{t}_{i}^{2}\right]
$$

Here $s$ can be interpreted as a measure of tracking defined as:

$$
\boldsymbol{s}=\dot{\tilde{\boldsymbol{\eta}}}+\lambda \tilde{\boldsymbol{\eta}}
$$

where $\lambda$ is a strictly positive constant which may be interpreted as the control bandwidth and $\eta_{d} \in C^{3}$ denotes the desired position and orientation of the vehicle. It is convenient to rewrite (28) as

$$
\boldsymbol{s}=\dot{\boldsymbol{\eta}}-\dot{\boldsymbol{\eta}}_{\boldsymbol{r}} \Rightarrow \dot{\boldsymbol{\eta}}_{d}-\lambda \tilde{\boldsymbol{\eta}}
$$

It is assumed that: $\boldsymbol{M}, \boldsymbol{C}(\boldsymbol{v}), \boldsymbol{D}(\boldsymbol{v}), \boldsymbol{g}(\boldsymbol{v})$ are linear in their parameters. This in turn suggests that we can apply the parameterization

$$
M \dot{v}_{r}+C(v) v_{r}+D(v) v_{r}+g(\eta) \triangleq \Phi\left(\dot{v}_{r}, v_{r}, v, \eta\right) \theta
$$

where $\boldsymbol{\Phi}(\cdot)$ is a known matrix and $\boldsymbol{\theta}$ is the unknown parameter vector. This parameterization requires that the variables: $v_{r}$ and $\dot{v}_{r}$ are computed according to the scheme of Fossen (1993), that is

$$
\begin{gathered}
v_{r}=J^{-1}(\eta) \dot{\eta}_{r} \\
\dot{v}_{r}=J^{-1}(\eta)\left[\ddot{\eta}_{r}-\dot{J}(\eta) J^{-1}(v) \dot{\eta}_{r}\right]
\end{gathered}
$$

Hence, the following theorem yields a convergent adaptive control law.

Theorem 2 (Convergent adaptive position/attitude control)

Consider the system (1), (2), and (3) and the following control law

$$
\begin{array}{lll}
\boldsymbol{u}_{d}=\boldsymbol{B}^{+}(\boldsymbol{v})\left[\boldsymbol{\Phi}\left(\dot{\boldsymbol{v}}_{r}, v_{r}, \boldsymbol{v}, \boldsymbol{\eta}\right) \hat{\boldsymbol{\theta}}-\boldsymbol{J}^{\mathrm{T}}(\boldsymbol{\eta}) \boldsymbol{K}_{\eta} s\right] ; & \boldsymbol{K}_{\boldsymbol{\eta}} \geqslant 0 \\
\boldsymbol{u}_{c}=\boldsymbol{u}+\hat{\boldsymbol{T}}_{d_{d}}-\boldsymbol{B}^{\mathrm{T}}(\boldsymbol{v}) \boldsymbol{J}^{-1}(\boldsymbol{\eta}) \boldsymbol{s}-\boldsymbol{K}_{u} \tilde{u} ; & \boldsymbol{K}_{u}>0
\end{array}
$$

The parameter estimates $\hat{\boldsymbol{\theta}}$ and $\hat{\boldsymbol{T}}$ are updated through the differential equations

$$
\begin{gathered}
\dot{\hat{\boldsymbol{\theta}}}=-\boldsymbol{\Gamma} \boldsymbol{\Phi}^{\mathrm{T}}\left(\dot{\boldsymbol{v}}_{r}, v_{r}, v, \eta\right) J^{-1}(\eta) s ; \quad \Gamma>0 \\
\dot{\hat{t}}=-\alpha_{i} \tilde{u}_{i} \dot{u}_{a i} ; \quad \alpha_{i}>0, \quad i=1 \ldots 6
\end{gathered}
$$


Then the signals $\tilde{\boldsymbol{\eta}}$ and $\tilde{\boldsymbol{T}}$ remain bounded and $\tilde{\boldsymbol{u}}$ and $s$ converges to zero as $t \rightarrow \infty$. In view of (28) this implies that $\tilde{\eta}$ converges to zero as $t \rightarrow \infty$. Special actions must be taken to avoid the singular point $\theta= \pm 90^{\circ}$.

Proof. The proof is found in Appendix B.

Remark 3 (Negligible actuator dynamics)

If the actuator is fast compared to the vehicle dynamics, it follows that $\boldsymbol{u}=\boldsymbol{u}_{\mathrm{c}}$ in view of $\boldsymbol{T}=0$. Hence, the Lyapunov function candidate (27) reduces to

$$
V=\frac{1}{2}\left[\boldsymbol{s}^{\mathrm{T}} \boldsymbol{M}_{\eta} \boldsymbol{s}+\ddot{\theta}^{\mathrm{T}} \boldsymbol{T}^{-1} \tilde{\theta}\right]
$$

which suggests that the adaptive control law should be expressed as:

$$
\boldsymbol{u}=\boldsymbol{B}^{+}(\boldsymbol{v})\left[\Phi\left(\dot{\boldsymbol{v}}_{r} \boldsymbol{v}_{r}, \boldsymbol{v}, \boldsymbol{\eta}\right) \tilde{\boldsymbol{\theta}}-J^{\mathrm{T}}(\boldsymbol{\eta}) \boldsymbol{K}_{\eta} s\right]
$$

with the parameter update law (35) to yield $V \leqslant 0$. This result is equivalent to the adaptive control scheme of Fossen and Sagatun (1991a,b) for underwater vehicles. Equation (38) was originally derived by applying the result of Fossen (1993) to the adaptive spacecraft attitude controller of Slotine and Benedetto (1990).

\section{Simulation study}

In the simulation study, we will consider two examples

- Adaptive speed control of a remotely operated vehicle (ROV)

- Adaptive autopilot for automatic ship steering.

Example 1. (Adaptive speed control of an ROV)

Healey and Marco (1992) propose that the ROV speed equation can be approximated as

$$
\left(m_{1}-X_{\dot{u}}\right) \dot{u}_{1}=X_{u|u|} u_{1}\left|u_{1}\right|+X_{n|n|} n|n|
$$

where $u_{1}$ is the surge velocity and $n$ is the propellor revolution. The system (39) is equivalent to the model

$$
m \dot{v}+d(v) v=u ; \quad d(v)=d_{o}|v|
$$

where $m=\left(m_{1}-X_{u}\right) / X_{n|n|}, d_{o}=-X_{u|u|}\left|X_{n|n|}, u=n\right| n$ and $v=u_{1}$. A normalized system with parameters $m=4$ and $d_{o}=1$ was used in the computer simulations. Furthermore, let the actuator dynamics be modeled as a first-order lag with time constant $T=1$, that is

$$
T \dot{u}+u=u_{c}
$$

where $u_{\mathrm{c}}$ is the commanded control input. In the computer simulation, the adaptive controller (Theorem 1) was sampled with a sampling rate of $10 \mathrm{~Hz}$. The state variables $v$ and $u$ and their corresponding tracking errors $\tilde{v}$ and $\tilde{u}$ are shown in Figure 1. The parameter estimates: $\hat{m}, \hat{d}_{o}$ and $\hat{T}$ and their true values are shown in Figure 2 . We see from the simulation that: $\tilde{v} \rightarrow 0$ and $\tilde{u} \rightarrow 0$ and that $\tilde{m}, \hat{d}_{o}$ and $\hat{T}$ converge to their true values in less than $50 \mathrm{~s}$. However, convergence of the parameters is only guaranteed if the external signal is persistently exciting (PE). 

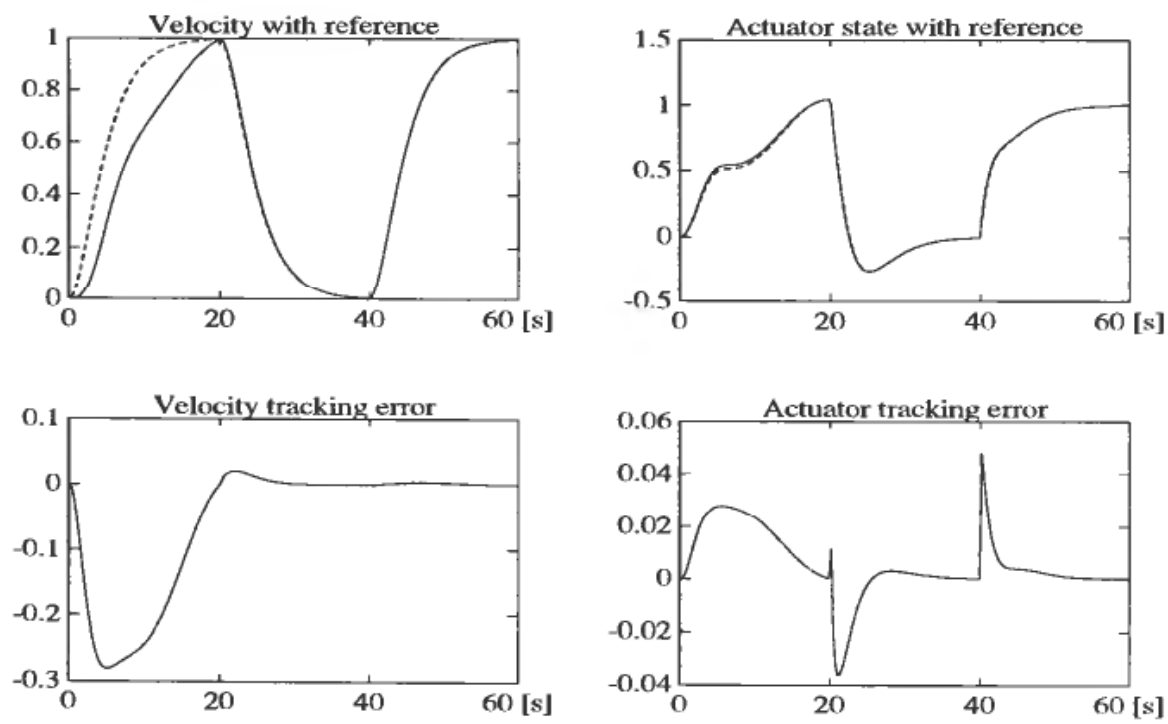

Figure 1. Upper plot shows the actual and desired surge velocity and actuator output while the lower plot shows their corresponding tracking errors (Example 1).
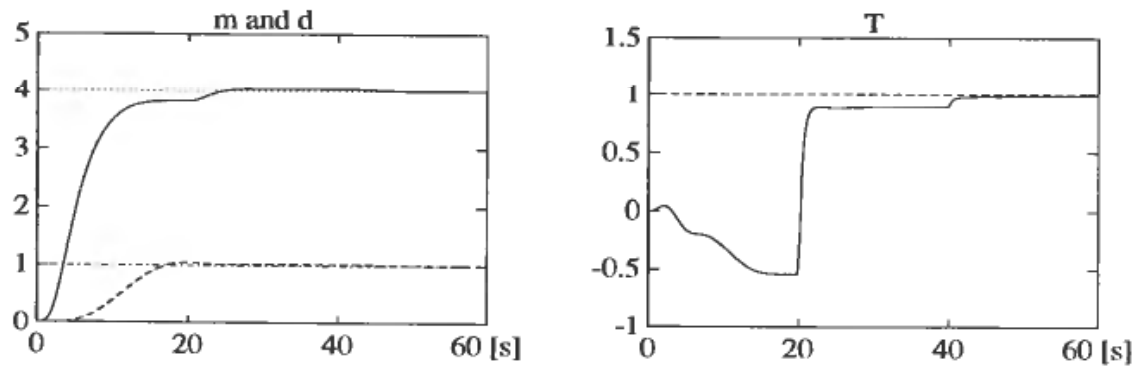

Figure 2. Normalized parameter estimates $\hat{m}, \hat{d}$ and $\hat{T}$ and their true values as a function of time (Example 1).

\section{Example 2. (Adaptive ship steering)}

Consider Nomoto's first-order model (Nomoto et al. (1957)) in the form

$$
T_{1} \dot{r}+r=K \delta ; \quad r=\dot{\psi}
$$

where $r$ is the heading rate, $\psi$ is the heading angle and $\delta$ is the rudder angle. $T_{1}$ and $K$ are the time and gain constant, respectively. Let the rudder dynamics be described by a first-order lag

$$
T \dot{\delta}+\delta=\delta_{c}
$$

where $T$ is the actuator time constant and $\delta_{c}$ is the commanded rudder angle. The system (42) and (43) is equivalent to the model

$$
\begin{aligned}
m \ddot{\eta}+d \dot{\eta} & =u \\
T \dot{u}+u & =u_{c}
\end{aligned}
$$

where $u=\delta, u_{c}=\delta_{c}, \eta=\psi, m=T_{1} / K$ and $d=1 / K$. A normalized system with parameters $m=4, d=1$ and $T=1$ is used in the computer simulations. As in the previous example, 

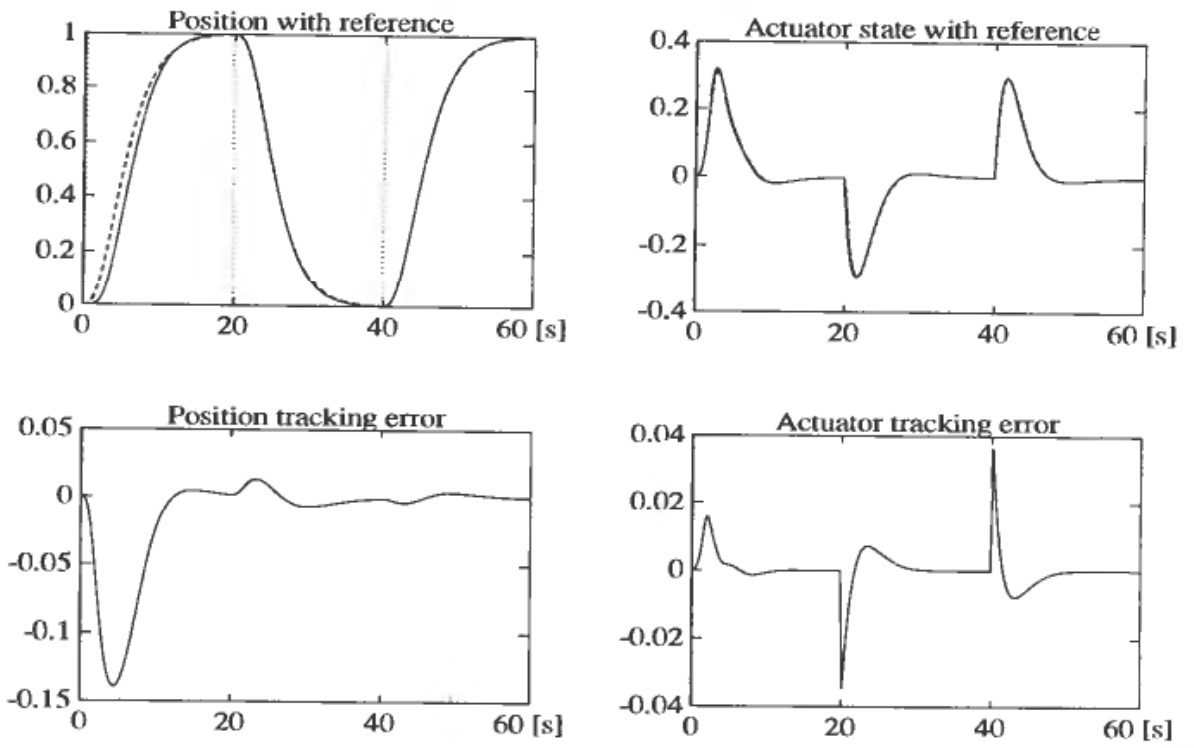

Figure 3. Upper plot shows the actual and desired heading angle and actuator output while the lower plot shows their corresponding tracking errors (Example 2).
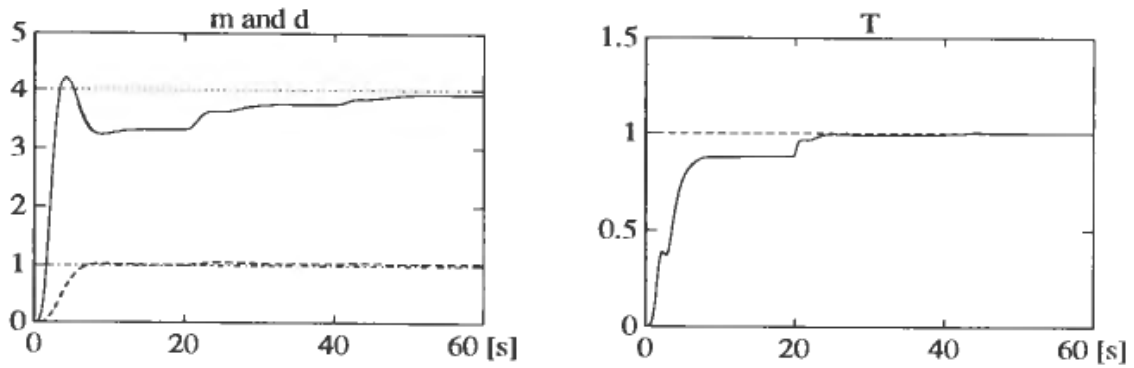

Figure 4. Normalized parameter estimates $\hat{m}, \hat{d}$ and $\hat{T}$ and their true values as a function of time (Example 2).

the adaptive controller (Theorem 2) was sampled with a sampling rate of $10 \mathrm{~Hz}$. The state variables: $\eta, u$ and their corresponding tracking errors $\tilde{\eta}$ and $\tilde{u}$ are shown in Figure 3 while the parameter estimates $\hat{m}, \hat{d}$ and $\hat{T}$ and their true values are shown in Figure 4. As in the first example, we see that the tracking errors converge to zero and that the parameters converge to their true values in less than 50 seconds.

\section{Conclusions}

A globally asymptotically stable adaptive controller for velocity control, and a convergent adaptive controller for position/attitude control of ocean vehicles in presence of significant actuator dynamics has been derived. The control laws exploit the physics and a priori information of the nonlinear vehicle dynamics and kinematics in terms of well known mechanical systems properties. In most control applications the dynamics of the actuator is neglected. Hence, parameter drift and robustness of the adaptive controller can present a severe problem. The new algorithm presented in this paper allows the designer to compensate for the vehicle and actuator dynamics in a systematic manner. The simulation study shows that excellent performance and robustness can be obtained both for an ROV speed control system and a ship stccring 
autopilot. For simplicity, the stability analyses presented in this paper utilize an actuator model described by a set of decoupled first-order systems with unknown time constants. It should be noted, that it is straightforward to extend the adaptive control scheme to a more general nonlinear actuator model:

$$
\dot{\boldsymbol{u}}+\boldsymbol{f}(\boldsymbol{u})=\boldsymbol{G}(\boldsymbol{u}) \boldsymbol{u}_{\mathrm{c}}
$$

where $f(u)$ and $G(u)$ are two unknown functions. If in addition we require that both functions are linear in their parameters, convergence of the tracking errors can be proven by small modifications of the proofs in Appendix A and B. Finally, it is quite obvious that the results presented in this paper are also valid for other types of mechanical systems, such as spacecraft, aircraft and robot manipulators.

\section{Appendix A}

\section{Proof of Theorem 1}

Differentiating (17) with respect to time and using the result from (9), yields

$$
\begin{aligned}
\dot{V}= & -\tilde{v}^{\mathrm{T}} \boldsymbol{D}(v) \tilde{v}+\tilde{v}^{\mathrm{T}}\left[B(v) u_{d}-\Phi\left(\dot{v}_{d}, v_{d}, v, \eta\right) \theta\right]+\dot{\tilde{\theta}}^{\mathrm{T}} \Gamma^{-1} \tilde{\theta} \\
& +\tilde{u}^{\mathrm{T}}\left[u_{c}-u-T \dot{u}_{d}+B^{\mathrm{T}}(v) \tilde{v}\right]+\sum_{i=1}^{6} \frac{1}{\alpha_{i}} \dot{\tilde{t}}_{i} \tilde{t}_{i}
\end{aligned}
$$

Substituting the control law (19) and (20) into this expression yields

$$
\dot{V}=-\tilde{v}^{\mathrm{T}}\left[K_{v}+\boldsymbol{D}(\boldsymbol{v})\right] \bar{v}+\left[\tilde{v}^{\mathrm{T}} \boldsymbol{\Phi}\left(\dot{v}_{d}, v_{d}, v, \eta\right)+\dot{\boldsymbol{\theta}}^{\mathrm{T}} \boldsymbol{\Gamma}^{-1}\right] \overline{\boldsymbol{\theta}}-\tilde{\boldsymbol{u}}^{\mathrm{T}} K_{u} \tilde{u}+\sum_{i=1}^{6}\left(\frac{1}{\alpha_{i}} \dot{\tilde{t}}_{i}+\tilde{u}_{i} \dot{u}_{d i}\right) \tilde{t}_{i}
$$

Finally, assuming that $\dot{\boldsymbol{\theta}}=0$ and $\dot{\boldsymbol{T}}=0$, suggest that the parameter adaptation laws should be expressed as in (21) and (22) to obtain

q.e.d.

$$
\dot{V}=-\tilde{v}^{\mathrm{T}}\left[K_{v}+D(v)\right] \tilde{v}-\tilde{u}^{\mathrm{T}} K_{u} \tilde{u} \leqslant 0
$$

\section{Appendix B}

Proof of Theorem 2

Differentiating (27) with respect to time and using Property 4 and 6 yields

$$
\dot{V}=s^{\mathrm{T}}\left[M_{\eta}(\eta) \dot{s}+C_{\eta}(v, \eta) s\right]+\tilde{u}^{\mathrm{T}} T \vec{u}+\dot{\ddot{\theta}}^{\mathrm{T}} \Gamma^{-1} \tilde{\theta}+\sum_{i=1}^{6} \frac{1}{\alpha_{i}} \dot{\bar{t}}_{i} \tilde{t}_{i}
$$

Substituting the system equations (25) and (3) into this expression and using the definitions (26) and (29) yields

$$
\begin{aligned}
\dot{V}= & -s^{\mathrm{T}} D_{\eta}(v, \eta) s+\left[J^{-1}(\eta) s\right]^{\mathrm{T}}\left[B(v) u-M(\eta) \dot{v}_{r}-C(v, \eta) v_{r}-D(v, \eta) v_{r}-g(\eta)\right] \\
& +\dot{\tilde{\theta}}^{\mathrm{T}} \Gamma^{-1} \tilde{\theta}+\tilde{u}^{\mathrm{T}}\left[u_{c}-u-T \dot{u}_{d}\right]+\sum_{i=1}^{6} \frac{1}{\alpha_{i}} \dot{\tilde{t}}_{i} \tilde{t}_{i}
\end{aligned}
$$

Since $s^{\mathrm{T}} J^{-1}(\eta) B(\eta) \tilde{u}=\tilde{u}^{\mathrm{T}} \boldsymbol{B}^{\mathrm{T}}(v) J^{-1}(\theta) s$, we can subtract $\boldsymbol{B}(\boldsymbol{v}) \tilde{\boldsymbol{u}}=\boldsymbol{B}(\boldsymbol{v}) \boldsymbol{u}-\boldsymbol{B}(\boldsymbol{v}) \boldsymbol{u}_{d}$ from the first bracket and add $B^{\mathrm{T}}(v) \boldsymbol{J}^{-1}(\eta) s$ to the second bracket. Applying the parameterization (30) yields

$$
\begin{aligned}
\dot{V}= & -s^{\mathrm{T}} D_{\eta}(v, \eta) s+\left[J^{-1}(\eta) s\right]^{\mathrm{T}}\left[B(v) u_{d}-\Phi\left(\dot{v}_{r}, v_{r}, v, \eta\right) \theta\right]+\dot{\tilde{\theta}}^{\mathrm{T}} \Gamma^{-1} \boldsymbol{\theta} \\
& +\tilde{u}^{\mathrm{T}}\left[u_{c}-u-T \dot{u}_{d}+B^{\mathrm{T}}(v) J^{-1}(\eta) s\right]+\sum_{i=1}^{6} \frac{1}{\alpha_{i}} \dot{\tilde{t}}_{i} \tilde{t}_{i}
\end{aligned}
$$


Substituting the control law (33) and (34) into this expression yields

$$
\begin{aligned}
\dot{V}= & -s^{\mathrm{T}}\left[K_{\eta}+D_{\eta}(v, \eta)\right] s+\left[s^{\mathrm{T}} J^{-\mathrm{T}}(\eta) \Phi\left(\dot{v}_{r}, v_{r}, \boldsymbol{v}, \eta\right)+\dot{\boldsymbol{\theta}}^{\mathrm{T}} \Gamma^{-1}\right] \tilde{\theta} \\
& \left.-\tilde{\boldsymbol{u}}^{\mathrm{T}} \boldsymbol{K}_{u} \tilde{\boldsymbol{u}}+\sum_{i=1}^{6}\left(\frac{1}{\alpha_{i}} \dot{\tilde{t}}_{i}+\tilde{u}_{i} \dot{u}_{d i}\right)\right) \tilde{t}_{i}
\end{aligned}
$$

Finally, assuming that $\dot{\boldsymbol{\theta}}=0$ and $\dot{\boldsymbol{T}}=0$, suggests that the parameter adaptation laws should be expressed as in (35) and (36) to obtain:

$$
\dot{V}=-s^{\mathrm{T}}\left[K_{\eta}+D_{\eta}(v, \eta)\right] s-\tilde{u}^{\mathrm{T}} K_{u} \tilde{u} \leqslant 0
$$

q.e.d.

Notice that global stability is guaranteed even for $\boldsymbol{K}_{\eta}=0$ since $x^{\mathrm{T}} D_{\eta}(v, \eta) x>0 \forall \eta, v, x \in \mathbb{R}^{6}, x \neq 0$ (Property 5).

\section{REFERENCES}

BARBÃLAT (1959). Systèmes d'èquations diffèrentielles d'oscillations non linèaires. Revue de Mathèmatiques Pures et Appliquèes, 4, 267-270. (in Frcnch).

Butler, H., Honderd, G. and VAn Amerongen, J. (1991). Reference model decomposition in direct adaptive control. International Journal of Adaptive Control and Signal Processing, $\mathbf{5}$, 199-217.

FJellstAD, O.-E., Fossen, T. I. and EGEL AND, O. (1992). Adaptive control of ROVs with actuator dynamics and saturation. In Proceedings of the 2nd International Offshore and Polar Engineering Conference (ISOPE), San Francisco, CA, June 1992.

Fossen, T. I. and SAGATUN, S. I. (1991a). Adaptive control of nonlinear underwater robotic systems. In Proceedings of the IEEE Conference on Robotics and Automation, pp. 16871695, Sacramento, California, April 1991.

Fossen, T. I. and SAGATUN, S. I. (1991b). Adaptive control of nonlinear systems: a case study of underwater robotic systems. Journal of Robotic Systems, 8, 393-412.

Fossen, T. I. (1992). Adaptive control of flexible joint manipulators. Submitted to the IEEE Transactions on Automatic Control.

Fossen, T. I. (1993). Comments on "Hamiltonian adaptive control of spacecraft". IEEE Transactions on Automatic Control, 38, 671-672.

Fossen, T. I. (1994). Guidance and control of ocean vehicles (John Wiley \& Sons Ltd., Chichester).

Healey, A. J. and MarCo, D. B. (1992). Slow speed flight control of autonomous underwater vehicles: experimental results with NPS AUV II. In Proceedings of the 2nd International Offshore and Polar Engineering Conference (ISOPE), San Francisco, CA, June 1992.

KIRCHHOFF, G. (1869). Ueber die Bewegung eines Rotationskorpers in einer Flussigkeit. Crelle's Journal, No. 71, pp. 237-273.

Newman, J. N. (1977). Marine Hydrodynamics (MIT Press, Cambridge, MA).

Nomoto, K., Taguchi, T., Honda, K. and HiRano, S. (1957). On the steering qualities of ships. Technical report, International Shipbuilding Progress, Vol. 4.

PoPOv, V. M. (1973). Hyperstability of Control Systems (Springer-Verlag, Berlin).

Sagatun, S. I. and Fossen, T. I. (1991). Lagrangian formulation of underwater vehicles' dynamics. In Proceedings of the IEEE International Conference on Systems, Man and Cybernetics, Charlottesville, VA, October 13-16, 1991, pp. 1029-1034.

Slotine, J. J. E. and Di BenEDETto, M. D. (1990). Hamiltonian adaptive control of spacecraft. IEEE Trans. Automatic Control, 35, 848-852.

Slotine. J. J. E. and LI, W. (1987). Adaptive manipulator control. A case study. In Proceedings of the 1987 IEEE Conf. on Robotics and Automation, Raleigh, North Carolina, pp. 1392-1400. 